\title{
Revista brasileira de geriatria e gerontologia: vários olhares sobre 0 envelhecimento
}

\author{
BrazilianJ armal of Gerontdyand Geriatrics severd viensonaging
}

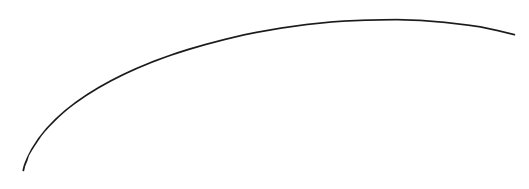

O crescente reconhecimento da importância da capacidade funcional como principal indicador na condução das ações de promoção, prevenção e atenção à saúde do idoso se reflete no temário dos artigos apresentados neste segundo número da Revista Brasileira de Geriatria e Gerontologia em 2011.

Entre os artigos originais, destacamos um grupo de pesquisadores da Universidade do Estado de Santa Catarina que analisou as diretrizes para implantação de programas de atividade física para idosos. A seguir, o Instituto Paulista de Geriatria e Gerontologia apresenta um estudo sobre o efeito de atividades de estimulação no desempenho de idosos em testes específicos.

A economia da saúde é outra área que tem crescido em importância, principalmente quando se atua com um contingente populacional no qual predominam as condições crônicas. A Universidade Católica de Brasília contribui com um estudo sobre o custo direto das fraturas de fêmur por quedas em idosos.

O envelhecimento bem-sucedido é um tema sempre atual, conforme aponta o estudo da Universidade Federal do Rio Grande do Sul sobre as aprendizagens no esporte competitivo, que foi avaliado como uma forma de adaptação de atletas que praticam corrida.

A hipertensão em idosos tem sido bem estudada no nosso país. Da Universidade Federal do Rio Grande do Norte vem a contribuição de um estudo sobre a função cognitiva e a capacidade funcional em idosos hipertensos, e da Universidade Estadual de Campinas vem outro estudo sobre hipertensão, intitulado "Hipertensão arterial sistêmica: aspectos clínicos e análise farmacológica no tratamento dos pacientes de um setor de psicogeriatria”.

A saúde bucal também está presente neste número, com um estudo da Universidade Estadual de Montes Claros sobre a autopercepção das condições bucais em uma população de idosos.

Outra questão de suma importância na área da saúde do idoso são os medicamentos potencialmente inapropriados para idosos e sua presença no SUS. Neste sentido, pesquisadores da Universidade Estadual de Maringá apresentam um estudo que avalia as listas padronizadas de medicamentos.

A osteoporose e o comportamento alimentar são tópicos especialmente importantes. Os fatores de risco da osteoporose na mulher idosa são estudados em outro artigo da Universidade Católica de Brasília. Os aspectos afetivos relacionados ao comportamento alimentar dos idosos 
frequentadores de um centro de convivência são analisados por um grupo de pesquisadores da Universidade de São Paulo.

A qualidade de vida de idosos é um tema bem desenvolvido neste número. Um grupo da Universidade Federal de São Paulo avalia a qualidade de vida de idosos atendidos em programa de assistência domiciliária, e o Instituto Paulista de Geriatria e Gerontologia apresenta um estudo sobre a cinesioterapia no tratamento da incontinência urinária em mulheres idosas, que também aborda qualidade de vida. A inclusão digital é outro tópico importante quando se pensa a qualidade de vida dos idosos. A associação entre percepção subjetiva de memória, habilidade manual e inclusão digital de idosos é estudada em interessante artigo proveniente da Pontifícia Universidade Católica do Rio Grande do Sul.

Quanto aos artigos de revisão, o cuidar está presente no estudo da Universidade Federal de Viçosa sobre o papel da família na tarefa do cuidar. Da Universidade Federal de Santa Maria e da Pontifícia Universidade Católica do Rio Grande de sul vem um estudo sobre o envelhecimento e longevidade no Rio Grande do Sul. E também apresentando uma revisão de literatura, a Fundação Comunitária de Ensino Superior de Itabira e a Universidade Fumec, Minas Gerais, contribuem com um estudo sobre as consequências das quedas em idosos vivendo na comunidade.

Por fim, mas não menos importante, há o relato de caso da Universidade Estadual Paulista sobre o treinamento com pesos como um modelo de programa sistematizado para idosos.

Portanto, em mais um número foi possível proporcionar a nossos leitores a visão com diversidade de temas relevantes sobre o envelhecimento, através de artigos provenientes das mais importantes instituições de todo o país. 